\title{
Image Synthesis Pipeline for Surface Inspection
}

\author{
Lovro Bosnar*, Doria Saric*, Siddhartha Dutta*†, \\ Thomas Weibel*, Markus Rauhut*, Hans Hagen ${ }^{\dagger}$, \\ Petra Gospodnetic* \\ * Image Processing Department, Fraunhofer ITWM, Kaiserslatuern, Germany \\ E-mail: lovro.bosnar@itwm.fraunhofer.de,doria.saric@helix.hr, s_dutta18@cs.uni-kl.de, \\ thomas.weibel@itwm.fraunhofer.de, markus.rauhut@itwm.fraunhofer.de,petra.gospodnetic@itwm.fraunhofer.de \\ $\dagger$ Computer Graphics and HCI Group, University of Kaiserslautern, Kaiserslatuern, Germany \\ hagen@cs.uni-kl.de
}

\begin{abstract}
Surface inspection development requires large amounts of image data representing the inspected product surface. The image data should contain both the ideal surface and the defective surface that can appear during production. Although image synthesis comes as a natural solution to this problem, its application is not straightforward in automated surface inspection environments. The reason for that is a lot of manual work that should be done for creating defects, simulating the inspection environment, and setting up the acquisition system for validation of simulation. To address these issues, we present a novel pipeline that automatizes surface defect creation, provides realistic rendering in a predefined inspection environment setup, and an acquisition system that enables comparison with the real images. The pipeline creates geometry-imprinted defects which combined with physically based rendering methods enable realistic light response for different light and camera positions during image synthesis. Finally, synthesized images can be compared with the real image taken in the same setup enabling verification. Also, synthesized images enable the visualization of visible surfaces and defects for a given inspection plan.
\end{abstract}

Index Terms-Mesh geometry models, Reflectance modeling, Simulation evaluation

\section{INTRODUCTION}

Industry 4.0 introduced the concepts of smart factories and smart manufacturing which are oriented towards automated production with high customization capabilities. Automated production further requires automated inspection of the products. Due to its high availability and applicability, visual inspection is a frequent inspection method [1]. Therefore, automated visual inspection, such as surface inspection, can be considered as an important part of quality assurance in the Industry 4.0 concept. Even though the inspection process itself is automated, development of one such inspection system requires an expert approach. For that purpose, research efforts have been focused on development of automated inspection planning tools, which can be used as an aid to experts developing surface inspection systems [1], [2]. The main goal of such planning tools is to find the optimal setup of acquisition hardware (camera and illumination) that ensure complete surface coverage of the product.

Good acquisition setup and development of robust image processing algorithms for defect detection are two highly

LEVIA'20: Leipzig Symposium on Visualization in Applications 2020 interdependent parts of inspection system design. Acquisition hardware is placed and calibrated to maximize the visibility of a defect, and the image processing algorithms are developed for images made with that particular acquisition setup. Therefore, development of automated surface inspection systems relies on a large amount of representative product image data. More specifically, image data containing as many defects as possible. Due to variety of reasons (frequency of defect occurrence, small production batches, etc.), the amount of available defects is frequently limited, which can cause problems in the development of robust detection algorithms. For that purpose synthetically generated datasets are needed. Those datasets would contain images of the product augmented with defects with a wide range of shapes and positions over the surface as they would appear in the real-world cases only after longer production periods.

Computer generated imagery (CGI) comes as a logical solution to the image synthesis problem, however, its utilization is not straightforward in the automated environments since it requires a lot of manual work done by experts. In this paper, we address those problems by offering a clear pipeline for realistic image synthesis of an object with both ideal and defective surface, as well as comparison to the imagery of physical objects for verification of the results. Synthesized images offer a way to visualise which parts of the surface and defects are visible for given inspection plan (i.e., camera and light positions). As it can be seen in Fig. 1, the pipeline consists of three modules. The first module is responsible for defect creation directly on object geometry. The second module performs physically based rendering of the object. The third module allows real image acquisition and therefore verification of the synthesized image.

\section{RELATED WORK}

Gospodnetic, Mosbach, Rauhut and Hagen [1] defined fundamental requirements for surface inspection planning pipelines and gave an example of a semi-automated visual inspection planning pipeline. The presented pipeline uses the geometry of the object for the purpose of viewpoint placement. The problem of data synthesis as a means to predict the inspection outcome has not been tackled, but the importance of 


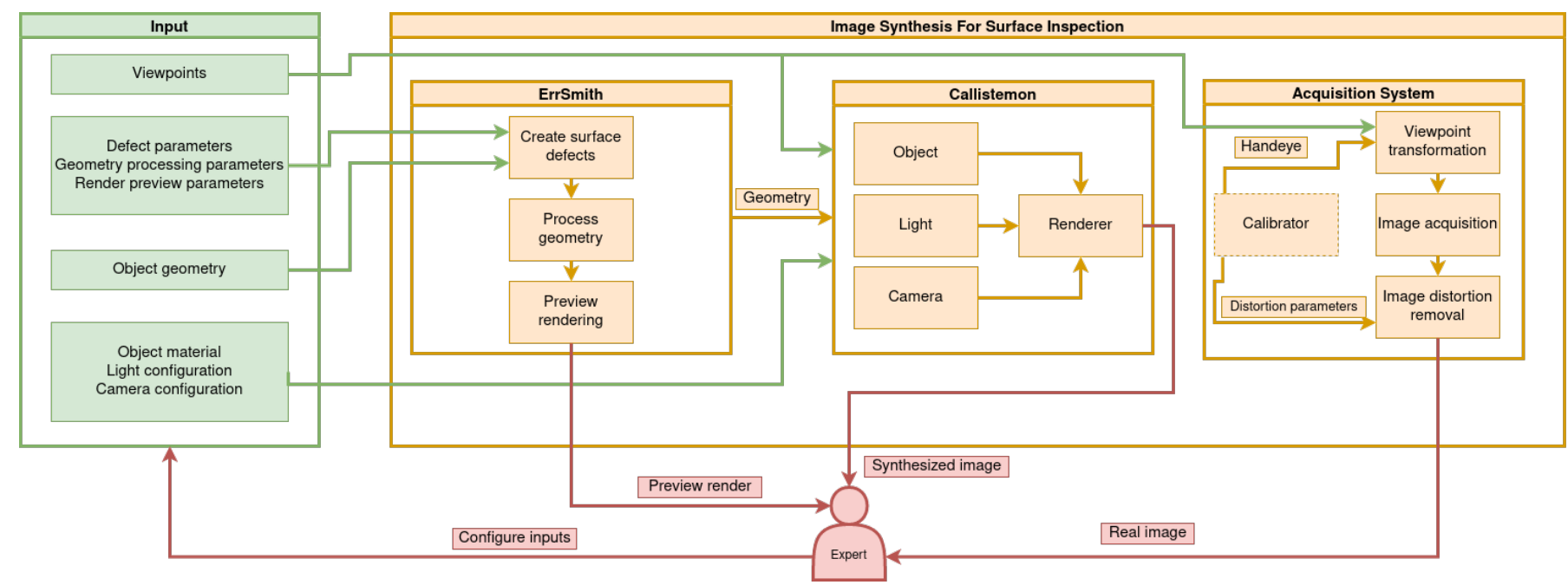

Fig. 1: Pipeline overview. Input data and its flow is represented in green color. Core pipeline and its modules are represented in orange color. Module outputs and expert interaction is represented in red color.

such work has been highlighted. The pipeline also introduced a verification system, used for coverage comparison, but the method used for correct positioning of the camera was not discussed.

Deficiency of defect data motivated defect image augmentation (Shorten and Khoshgoftaar [3]). Standard image transformations are used to produce more data, which in some respects becomes redundant or repetitive, but makes the detection robust to defect position, size, etc. Autoencoder and GANbased solutions can infill defect-free images with synthesized defects learned on real defect images (Tang, Yang, Xiong and Yan [4]). However, the learning process is unstable without larger amounts of data and requires a confident validation for generated images. Even so, it could only be applied to simple geometries in ideal inspection lightning conditions.

As an alternative, Merillou, Dischler and Ghazanfarpour [5] propose a hybrid approach to effectively render surface scratches. They couple bidirectional reflectance distribution functions (BRDFs) with texture mapping. However, it involves taking measurements of real scratches and the profile curve of the defect before mapping it to the model. Desbenoit, Galin and Akkouche [6] built an atlas of template fracture models imitating real cracks on various materials. The fracture model is automatically mapped onto the 3D model and carved into object surface using the Boolean difference operator. Although lightweight, it is still time consuming.

Haindl and Filip [7] provided taxonomy of the reflectance models, highlighting the scale of the observation as an important aspect. BRDF represents an efficient model for homogeneous surfaces (i.e., surfaces seen from a larger distance). Ngan, Durand and Matusik [8] evaluated analytical BRDFs in terms of their ability to fit measured bidirectional distribution function data. They concluded that microfacet based models (Walter, Marschner, Li and Torrance [9]) provide a faithful, physically based appearance.
Mohammadikaji [2] and Reiner [10] tackled with realistic image synthesis for machine vision inspection systems. Mohammadikaji coupled ray tracing with Fourier optics methods for introducing wave optics effects. While it produces good results for laser based illumination, in case of white light illumination Dong, Walter, Marschner and Greenberg [11] concluded that same result can be achieved using geometric optics models which are widely available in existing physically based rendering engines. Therefore, this approach lacks generality. Reiner [10] proposed more general simulation approach but lacks clear pipeline.

Evaluation of realism in photorealistic rendering has been oriented towards appearance and was used to assess the subjective experience of a viewer (Kolivand, Sunar, Kakh, AlRousan and Ismail [12], Greenberg et al. [13]). The reason likely lies in the fact that recreating the same environment is a challenging process. Recently, Stets et al. [14] introduced a reassembly and multimodal digitization pipeline containing a controlled environment to precisely capture a given scene, obtaining almost pixel-precision when comparing simulated and captured images.

Obtaining correct camera setup involves camera calibration and hand-eye calibration. Camera calibration determines relative orientation of the camera to a calibration object and also its internal parameters, while hand-eye calibration determines the rigid transformation from robot end-effector to camera coordinate system. For hand-eye calibration, Tsai and Lenz [15] suggested a star-like viewpoint pattern while acquiring checkerboard images. Pachtrachai, Allan, Pawar, Hailes and Stoyanov [16] suggested calibration without markers. The downside of this approach is the lack of accuracy. Antonello, Gobbi, Michieletto, Ghidoni and Menegatti [17] and Wang, $\mathrm{Lu}, \mathrm{Hu}$ and $\mathrm{Li}$ [18] proposed the approach in which the robot automatically takes images of the calibration object and performs hand-eye calibration. Recently, Lyngby, Matthiassen, 
Frisvad, Dahl and Aanæs [19] used hand-eye calibration algorithm developed by Liang and Mao [20] for accurately aligning the camera position and orientation of the robot to measure the BRDF. None of those approaches discuss cameras with a narrow field of view, which is essential in the most surface inspection scenarios.

\section{REQUIREMENTS ANALYSIS}

CGI techniques such as [21], [22], [23], [24] are commonly used for generation of photo-realistic images and are capable of producing remarkable results. However, their practical use in the field of surface inspection is minimal [2], [10]. We assume that the explanation lies in the following reasons. Firstly, defects can occur in a wide range of shapes and positions over the surface introducing uncertainty which must be dealt with. Secondly, CGI techniques are developed with the goal of providing high flexibility and freedom for artists. Artists then manually create scene elements such as objects, materials, lights etc. This makes the direct usage of such techniques not applicable for environments which aim towards automation (e.g., surface inspection development). Finally, confidently using synthesized images for representing the real physical objects requires an acquisition system followed by verification methods that can prove the quality of the object properties in the synthesized image. Guided by the aforementioned reasons, we define three main requirements which should be satisfied by a defect synthesis pipeline:

R1 Must be capable of producing defects of various shapes and positions over the surface of the object.

R2 Must produce realistic images of the object.

R3 Must compare the synthesized image with a corresponding image acquired in the real world on the spot.

\section{Pipeline FOR IMAge Synthesis}

As mentioned in Section I, the presented pipeline consists of three main modules shown in Fig. 1:

- ErrSmith, discussed in section IV-A, creates defects directly on the $3 \mathrm{D}$ model of the object based on the provided defect parameters.

- Callistemon, discussed in section IV-B, incorporates the object material and geometry as well as light and camera properties used in surface inspection to reconstruct a 3D scene resembling the surface inspection environment. The 3D scene is then rendered from different camera viewpoints.

- Acquisition system, discussed in section IV-C, incorporates a semi automatic hand-eye calibration and camera calibration as well as automatic image acquisition of the real images taken from the same viewpoint as in Callistemon. Images acquired using the acquisition system enable verification of the synthesised images.

\section{A. Errsmith}

Computer graphics artists often spend a lot of time on each 3D model, sculpting surface irregularities and introducing color modification to make the object seem more real. To automate defect creation, we developed ErrSmith, a module which can automatically make geometrical surface defects on any given 3D model based on a provided set of parameters. For preview purposes, it is possible to render images of the object containing virtual defects. ErrSmith consists of defect creation, geometry processing, and preview rendering (see Fig. 1). It can be easily applied or adapted to various conditions and use cases.

1) Defect Creation: For the purpose of this work, defects are considered to be irregular indentations of varying sizes on the surface of the object (i.e., dents and scratches). Therefore, first a defect tool is made. It is a smaller 3D object manually modelled to resemble a $3 \mathrm{D}$ negative of defect representation on the surface, as can be seen in Fig. 2. The tool is then partly imprinted into surface of a given 3D model. Tools can be smooth and regular or quite complex with rough surfaces. The variety and complexity of defect shapes depends on the variety and complexity of the defect tools.

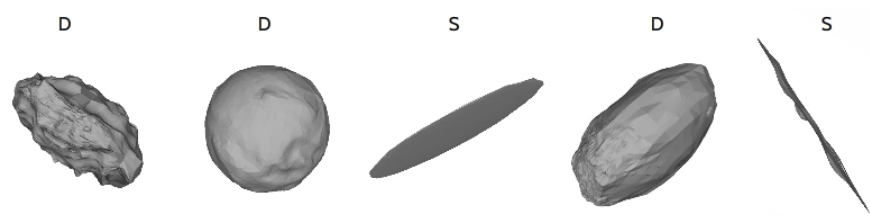

Fig. 2: Examples of some defect tool models. The ones with the label $\mathbf{D}$ are creating dents, while the very thin ones below $\mathbf{S}$ labels are used for scratches (sharp tools imitating a saw or a blade).

The input parameters for ErrSmith define defect types, number of defects (per type or in total), and defect sizes. All of these parameters can be set to fixed values or defined as a range of values from which the value should be sampled.

The following steps are repeated for each defect that should be made on the object:

1) Select a random defect tool belonging to the tool collection of the chosen defect type

2) Randomly translate the defect tool anywhere on the grid

3) Scale the tool with defect size parameter

4) Randomly rotate the defect tool

5) Translate the origin of the tool to an arbitrary point on the closest face of the object

6) Apply the Boolean difference with the object mesh to create a geometrical surface defect

2) Geometry Processing: After creating defects, further geometry manipulations can enhance the look of rendered images. Some simple-geometry models require processing before being rendered. For example, we could add some noise to face normals to simulate a rough surface. With simple geometries, all the faces first need to be subdivided by a certain factor. Otherwise, the model will have an insufficient number of normals to simulate surface roughness. In addition to that, edge roughness can be achieved by selectively adding some noise to vertex normals. 
3) Preview Rendering: Lightweight, preview rendering developed using Pyrender, offer a quick way to visualise ErrSmith results. Depending on the rendering parameters (camera setup, lights, viewpoints, number of images), a preview is rendered. For one given viewpoint, the preview includes a synthesized image of the defective object and a capture of the defect map. Defect map is a 3D model of that same object, with defective areas colored white and the rest in black. The captures of the defect map mark exact defect positions on the virtual image. An example of a preview can be seen in Fig. 3. If the rendering parameters were odd and the defects were hardly visible on the synthesized image, one could check the binary image to notice them. Additionally, the binary 2D images of the defect map could be used as the defect labels for the synthesized images in deep learning applications.
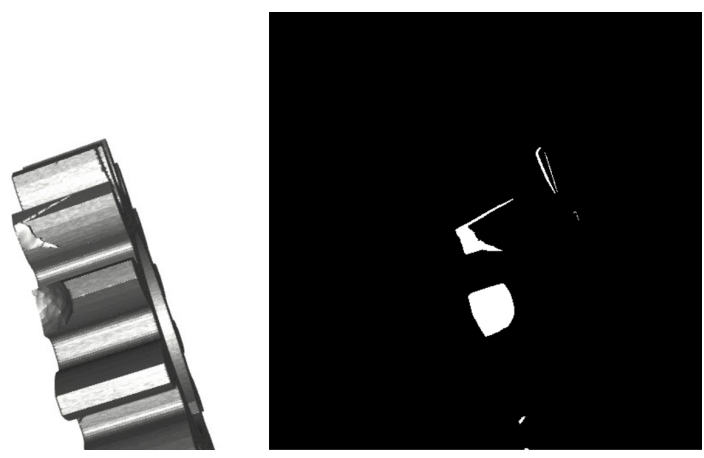

Fig. 3: Preview of defects created on the model of a gear. Virtual image with the defects (left) and the corresponding defect map capture (on the right).

\section{B. Callistemon}

Once the defects are introduced on the object geometry, synthesis of realistic images, similar to those acquired by a visual surface inspection system, is done using Callistemon. Callistemon serves as a connection between the surface inspection planning data and a physically based rendering engine. Therefore, it offers a way of visualising defected geometry with physically based material in an simulated environment. Surface inspection planning data consists of an object geometry and viewpoints which define camera placement and orientation, relative to the given object. A viewpoint is defined as a $9 D$ vector $\mathbf{v}=[\mathbf{p}, \mathbf{d}, \mathbf{u}], \mathbf{p}, \mathbf{d}, \mathbf{u} \in \mathbb{R}^{3}$. $\mathbf{p}$ represents a point in space where the camera is positioned, $\mathbf{d}$ is the direction of the optical axis of the camera and $\mathbf{u}$ represents orientation of the camera around the optical axis. In order to synthesize an image from a given viewpoint, a digital representation of the inspection environment (scene) is required. The scene consists of a light source, a camera and an object, each of which is described by a set of inputs. Required inputs are object mesh and viewpoints. Optional inputs are object material, light source geometry and material, environment parameters and camera parameters. To systematize the rendering process optional inputs have default values, but depending on application can be adjusted.
1) Object, Camera and Light Parameters: To simulate the appearance of the object during inspection, its geometry and material specifications are required. The geometry should be a realistic mesh representation of the object. Material specification is separated into reflectance properties and spatial variation details (i.e., texture). Realistic reflectance properties are described using physically based BRDFs available in Callistemon's rendering engine as Open Shading Language (OSL) closures [25]. User can choose the BRDF by choosing the OSL closure and specifying its parameters. Those BRDFs are resulting in perfectly smooth surface as viewed from a large distance. Therefore, they contain no spatial variation. Spatial variation in synthetic images is a result of color and surface roughness variation and is specified using procedural texturing. The user can describe the variation using OSL. Description accounts for variation which is much smaller than the size of the object, but much larger than the light wavelength [21]. Therefore, smaller scale of variation should be defined using BRDFs, while the larger scale of variation should be present in object geometry.

Simulating inspection environment requires that light source can be positioned relative to the camera or relative to the object. Defining the light relative to the camera will change its position in the scene for each new camera position. Otherwise, if the light position is defined relative to the object, its position $\mathbf{p} \in \mathbb{R}^{3}$ must be provided in object coordinate space and will remain the same for all the viewpoints. Callistemon supports physical (area) lights which are prerequisite for physically based simulation. Physical lights have certain shape and emit light from the surface of the shape. Therefore, physical light requires geometry and material specification. Light geometry should match the geometry of the real light because its area greatly influences the total amount of light in the scene. Emission material properties should be defined by the user using OSL (e.g., amount and color of light). The camera is simulated using a pinhole camera model and a set of parameters describing the real camera. The parameters include resolution, film dimension, pixel size and focal length.

\section{Acquisition System}

To enable validation of the synthesized images, it is necessary to obtain the real (ground truth) images in the same physical environment. Defining the environment for a surface inspection validation system is not as complex as for real life scenes (e.g., outdoor) since inspection system requires precise and consistent imaging conditions. Image acquisition conditions in an inspection system are determined by object placement, one or more cameras and one or more illumination devices. Object placement process determines the way in which the object is placed into the inspection system and manipulated during the inspection (e.g., manual placement, conveyor belt, turntable, manipulator device, etc.). Camera is always positioned relative to the object and represented by a single viewpoint from which an inspection image is acquired. Illumination devices can be relative to a camera or fixed relative to the object. Fixed illumination devices will 
have the same position regardless of the viewpoint which is used to acquire an image and can be used with more then one viewpoint, while relative illumination devices depend on the camera position and are typically only used by that single viewpoint.

An acquisition system provides a possibility to compare synthetic images to a precisely determined environment in an automated, consistent and reproducible manner. For that purpose we assume that the positioning system in an actual inspection system will always place the object at the same position. With such assumption it is possible to precisely define the position of the object within the acquisition system. The camera is attached to a manipulator device, thus enabling both positioning precision and repeatability. The illumination device position can either be fixed or attached to the same manipulator device as the camera. Finally, the system is given a list of camera viewpoints defined in Section IV-B, which are reached using the manipulator device.

Synthetic and real images are created independently, therefore, it is not possible to rely on any kind of markers, like it has been done by Stets et al. [14]. Instead, the precise positioning of the acquisition hardware within the scene requires the application of calibration techniques. The manipulator device carrying the camera is 6 degrees of freedom robotic arm. The camera is attached to its end-effector, but the coordinate systems of the robotic arm and the camera do not coincide. Therefore, moving a camera to a precise position using a robotic arm requires hand-eye transformation describing the transformation between the end-effector coordinate system and camera coordinate system [15]. Camera is fix-mounted to the end-effector, therefore the hand-eye transformation does not change during the acquisition process, but should be recalculated with any change in the acquisition system (e.g., focal length of the existing camera). Further, it should be recalculated regularly to account for the wear and tear effects.

We introduce an semi-automatic calibration approach. First, for intrinsic calibration, images with varying viewing angles of the checkerboard which is fixed in the scene are automatically acquired. Narrow field of view at the focusing distance and large aperture cause shallow depth of field, therefore some acquired images above the pattern can not capture the checkerboard clearly. As a solution, the checkerboard is fixed in the robot space. Various boundary coordinate ranges within the robotic coordinate system are defined. The range consists of the edge points from where the full checkerboard is in view to the points where the checkerboard corners are no longer in the view. Spanning the robot hand through these ranges allows to capture different views of the calibration object. For an accurate calibration, more than 3 boundary coordinates are specified. The system requires the number of images to be acquired for the calibration, which are then taken by the robot hand striding over boundary coordinates. Calibration is sensitive to exposure which affects the detection of corners. Therefore, images without all corners detected are automatically rejected by the system. The same images are then used for hand-eye calibration. For each image the transformation of the calibration pattern to eye can be estimated from 3D-2D correspondences using Lavenberg-Marquardt optimization as done by Antonello, Gobbi, Michieletto, Ghidoni and Menegatti [17]. This approach is used for calculating the hand-eye transformation. Since the robot knows the transformation from base to end-effector, the hand-eye transformation can be calculated using the calibration method provided by Tsai and Lenz [15]. Schematic representation of the manipulator is presented in Fig. 4. After finding the hand-eye transformation, any point in camera space can be converted to end-effector space using:

$$
\left[\begin{array}{lll}
X_{e} & Y_{e} & Z_{e}
\end{array}\right]^{T}=H_{c e}\left[\begin{array}{lll}
X_{c} & Y_{c} & Z_{c}
\end{array}\right]^{T}
$$

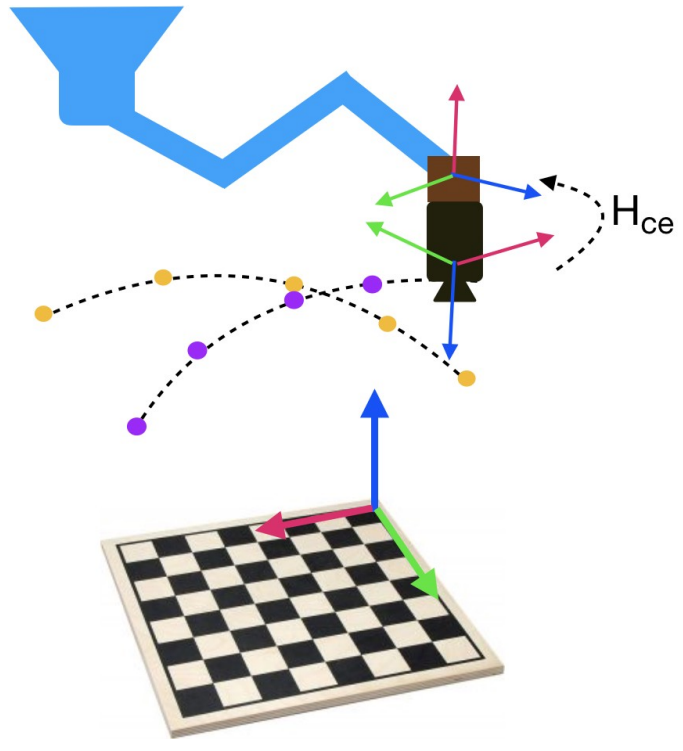

Fig. 4: Schematic representation of how the camera is moved by the robot end-effector while taking images of the checkerboard at different positions. The yellow and the purple dots show the positions calculated by the acquisition system at runtime from the given boundary coordinate ranges. $H_{c e}$ is the resulting matrix for camera coordinate system to end-effector coordinate system transformation.

\section{Results}

\section{A. Producing Defects}

ErrSmith automatizes the process of modeling surface defects. There are two main types of surface defects applicable in it - scratches and dents. For each type of defect, a collection of custom defect tool models was made (both from the sphere model). All the tools are of uniform size and made with Blender, which has advanced sculpting features widely used among computer graphics artists. The examples of defect tools in Fig. 2 were specifically used on the gear model in Fig. 5 to obtain artificial defects which can be seen in the Fig. 8. On the central image, we can see how the first defect tool damaged a gear tooth. When running ErrSmith module, defined parameters were specified as in Table I.

This assumes ErrSmith will make between 1 and 8 scratches and 2 to 5 dents. Scratches will have arbitrary size from 0.1 


\begin{tabular}{l|l|l}
\hline \multirow{3}{*}{$\begin{array}{l}\text { Defect } \\
\text { creation }\end{array}$} & defect type & {$[$ 'scratch', 'dent'] } \\
\cline { 2 - 3 } & defect size & {$[(0.1,0.35),(0.2,0.4)]$} \\
\cline { 2 - 3 } & number of defects & {$[(1,8),(2,5)]$} \\
\cline { 2 - 3 } Geometry & defect coordinates & - \\
\hline \multirow{2}{*}{\begin{tabular}{l} 
processing \\
\cline { 2 - 3 }
\end{tabular}} & face normals noise & Gauss $(0,0.001)$ \\
\cline { 2 - 3 } & vertex noise & - \\
\cline { 2 - 3 } & face subdivision & 2 \\
\hline
\end{tabular}

TABLE I: ErrSmith parameter values for the images in Fig. 8.

to 0.35 . Dents will be large between 0.2 and 0.4 . Gaussian noise will be added to the face normals and all the faces will previously be subdivided by factor 2 . There is an option to specify the number and size of defects in total (instead of separately per each defect type). For some specific use cases, the precise defect locations can be defined. The user can also specify the path to defect tool models, which enables the user to directly influence the defect appearance by modeling his own tools.

The outputs from ErrSmith, regardless of whether the images are being rendered for the preview, involve: (1) an STL file containing the defective object geometry, (2) an OBJ file containing a defect map in 3D, and (3) a JSON file containing metadata. Metadata includes all the parameter values (either default or defined by the user) for defect creation and geometry processing, paths to exact defect tools with exact defect locations they were applied to, and the amount of time it took to produce each defect.

\section{B. Realistic Image Synthesis}

Callistemon has been used to recreate surface inspection environment and render an object within it from the provided camera viewpoints. The scene consisted of the metal gear object and the physical light source (see Figures 5 and 6).

Object geometry was provided with the real object. Material property was configured using metal-specific physically based microfacet model (i.e., metalBRDF) provided in Appleseed [22]. Roughness value was set to 0.25 . Anisotropy amount was set to 0.7. Fresnel normal incidence color was set to $(196,199$, 199) sRGB. Spatial variation was introduced using procedural texture in OSL defined using:

$$
v(x, y)=\sqrt{(x+c)^{2}+(y+c)^{2}} \bmod d
$$

Where $c=S(r \bmod 1.0), r=\sqrt{x^{2}+y^{2}} . S(\cdot)$ represents smoothstep function, $d \in \mathbb{R}$ is used for controlling pattern density, $x$ and $y$ are coordinates of the shading point $P \in \mathbb{R}^{3}$. Value of the function is multiplied with the calculated color of metal material. The resulting render is in Fig. 6.

Camera and light source properties were determined based on the acquisition system described in Section V-C. The light source geometry was created using Blender (see Fig. 5). Light source material was set to diffuse emissive distribution function. Light source position is defined relative to the camera. Callistemon allows configuration of the environment light color and intensity. As the environment influence is negligible in the real setup, black, non-emitting environment is used in
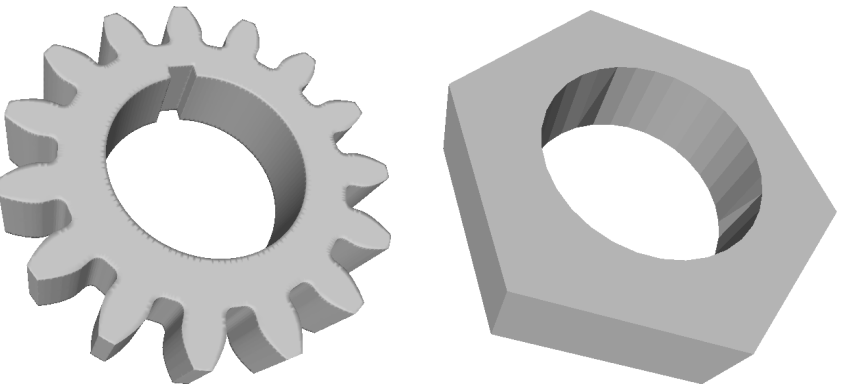

Fig. 5: Geometry of the object (left) and light (right) used in our surface inspection environment.

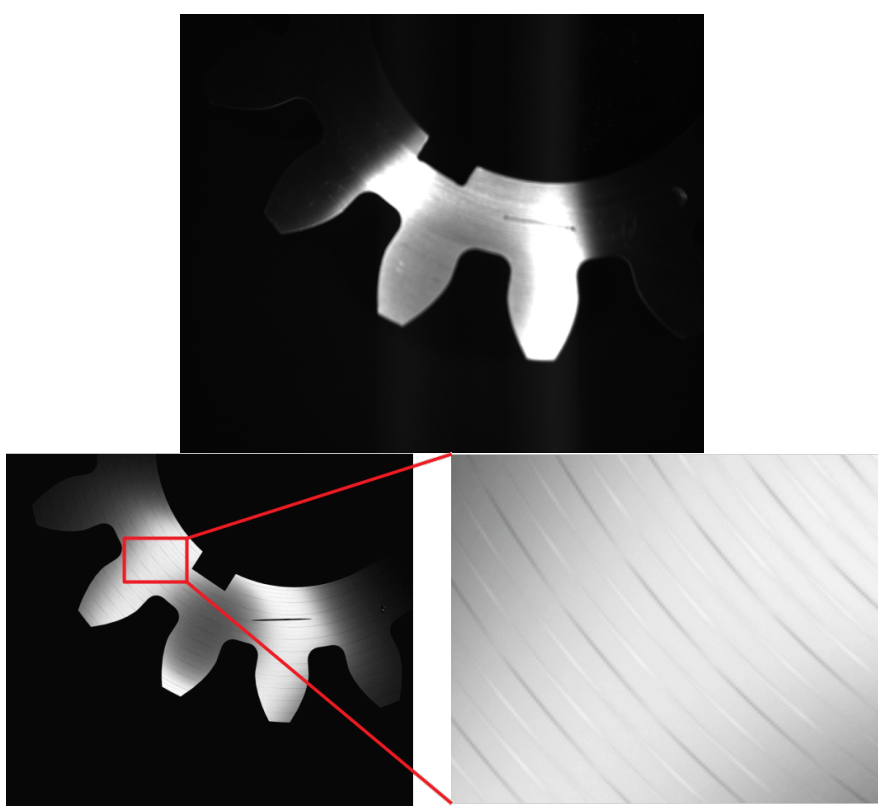

Fig. 6: Top: real image acquired by acquisition system. Bottom: rendered image with metal material and texture which is also magnified on the right. Note the defective geometry which was introduced using ErrSmith.

the simulation. Callistemon uses Appleseed, an open-source, physically based global illumination rendering engine. Modern light transport technique, unidirectional path tracing, was used along with five passes and adaptive pixel sampler.

Binary mask (see Fig. 7) of the object was created without any additional input. First, constant color was applied to the object. Next, environment light was enabled. These steps allow for a clear boundary between the object and the environment. Finally, using OpenCV [26] binary thresholding was performed on the rendered image. Due to discarded shading information, parts of the object that are not seen in realistic render are seen in binary image (e.g., inner side of the gear object).

Defective geometry created by ErrSmith was used in Callistemon (see Fig. 8). Beside defects, rendered images contain metal material as described above. 

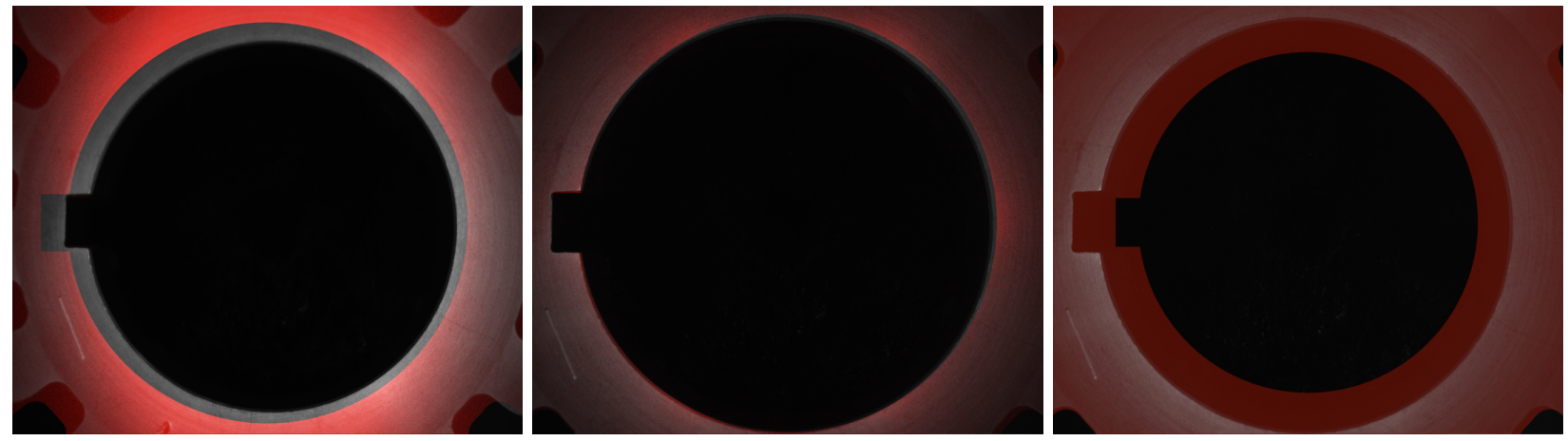

Fig. 7: Left: overlap of rendered (red object) and real image without calibration. Middle: overlap of rendered (red object) and real image with calibration. Right: overlap of binary mask (red object) and real image with calibration.
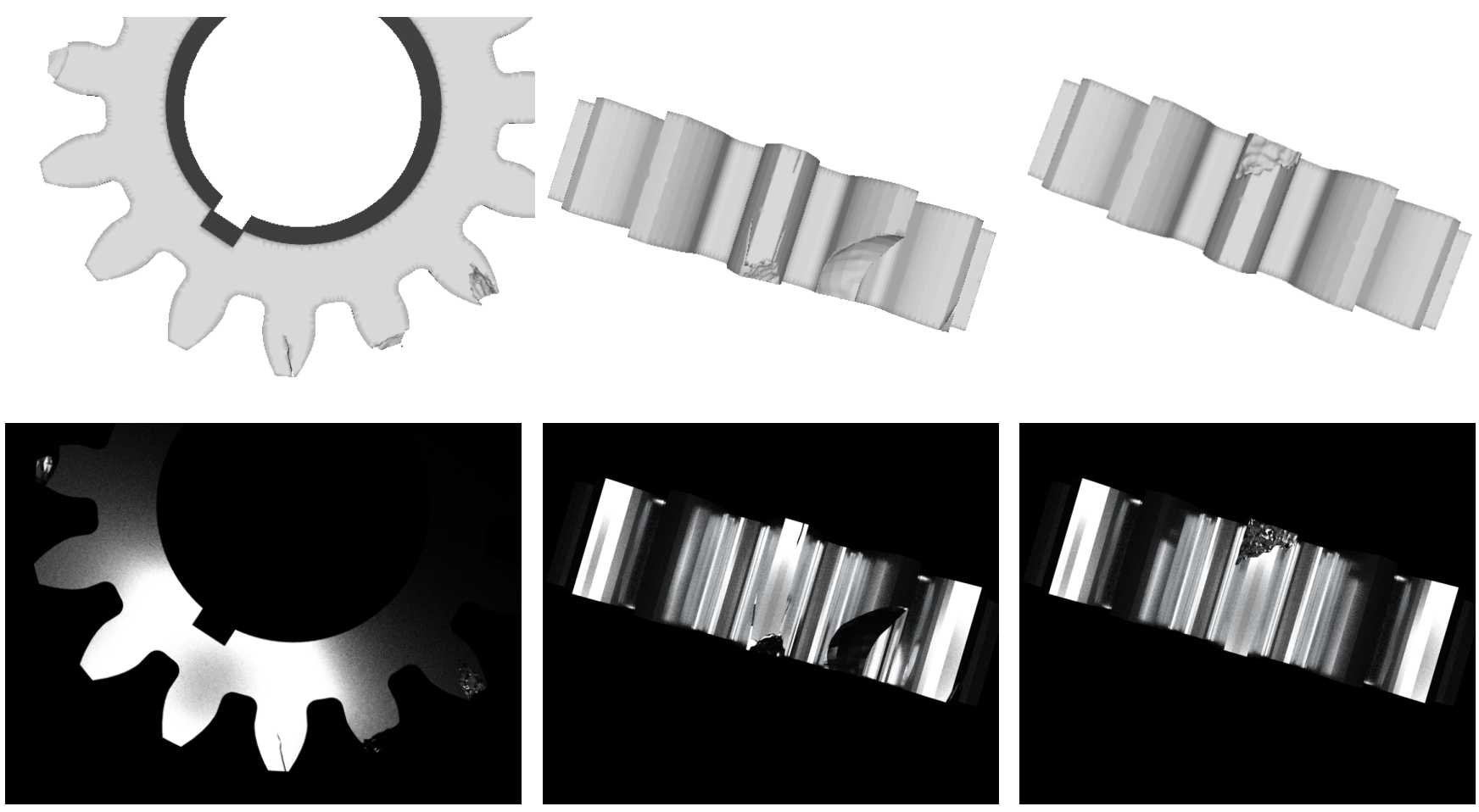

Fig. 8: Top row: defective geometry created using ErrSmith. Bottom row: synthesized images using Callistemon. Images show visibility of surfaces and defects for particular light and camera setup. Some scratches are deep therefore light is trapped. Dents show complex light response due to complex internal structure.

\section{Acquisition System Setup}

For the purpose of this work, both the camera and the illumination device have been mounted on a UR3 robot. The camera was Prosilica GC 2450 with a Sony ICX625 CCD sensor - an industrial, grayscale camera with mounted Kowa $12 \mathrm{~mm}$ lens. The sensor has resolution of $2448 \times 2050$ pixels with $3.45 \mu \mathrm{m} \times 3.45 \mu \mathrm{m}$ pixel dimensions. The camera had a fully opened fixed aperture and focusing distance set to 146 $\mathrm{mm}$. The illumination device is a DCM Sistemes ALU1006A630C ring light mounted around the camera lens.

1) System Configuration: A checkerboard pattern was used to perform the hand-eye calibration. Due to shallow depth of field present in the camera setup, checkerboard with $11 \times 9$ squares sized $6.6 \mathrm{~mm}$ was used. Calibration accuracy is limited to the accuracy of the printed checkerboard, while an error by a millimeter can produce large pixel deviation in the image. As discussed in section IV-C it is required to define boundary coordinates for the robotic arm. To determine each boundary range the UR3 teach-in pendant was used. Because the boundary coordinates are manually inputted to the system, the target reachability is satisfied. To achieve low error, a number of different viewpoints were used while striding over boundary coordinates. 9 different boundary coordinates are calculated using the UR3 teach-in pendant and a total of 80 images from 
different viewpoints were requested to be captured. Based on that the system calculates 80 different positions from which images will be captured. Each of these positions is then randomly mixed and the robot takes one image for each of these positions. To ensure collision avoidance, the robot was made to return to a safe position before going to take the next position. To detect corners automatically OpenCV [26] and ViSP [27] libraries were used. Images without all corners detected corners are automatically rejected by the system. The rejection rate is lower when the images are of lesser resolution compared to $100 \%$ resolution. This is due to the fixed size of corner detection filters in OpenCV, where the convolution size is too small to detect corners in high-resolution images.

\section{Intrinsic and Hand-eye Calibration}

The implementation of the intrinsic and hand-eye calibration has been provided by ViSP [27]. The final output of the process is a $4 \times 4$ hand-eye matrix $H_{c e}, 3 \times 3$ calibration matrix $K$, and the radial distortion parameter $k_{1}$. These details are used by the acquisition system. $k_{1}$ is used for undistorting the images. To validate the hand-eye transformation, known 3D points in robot space are back-projected into the 2D image captured by the camera. In a robust hand-eye estimate, the back-projected 3D points coincide with the seen 2D points in the image.

\section{DISCUSSION}

The resulting images in Fig. 8 show us how using just 8 defect tool models in total can produce a complex variety of surface irregularities. Since the tool is partly carved into the object, there is a great number of possibilities even for one defect tool, depending on how the tool is positioned, scaled, or rotated. Applying Boolean operator with complex defect tools allowed us to introduce more complexity and diversity into defects, while remaining computationally inexpensive. This shows that our pipeline satisfies the requirement R1. However, some faster and optimized Blender-external resources should be used for these operations in the future. Introducing more defect types would enable new use cases (e.g., color modifications imitating stains). Geometry imprinted defects allow using arbitrary material during image synthesis, since defects are part of the mesh and not the material. Also, this way defects behave correctly under arbitrary light and camera positions. Additionally, there is a space for improvement of defect tool models by experimenting with different modelling techniques or other geometries. Applying physically based material and texture enabled more realistic appearance of the synthesised object as it can be seen in Fig. 6. Therefore, requirement $\mathbf{R 2}$ is satisfied. Combination of physically based material and modern light transport results in accurate reflections and realistic appearance. Also, due to roughness, certain parts of the object are visible more clearly while other parts are not visible at all (Fig. 8). This is important for surface inspection tasks, because viewpoint coverage in this case can be lower than coverage estimated purely using the geometry. Clearer contrast can be achieved using advanced light distribution description such as Illuminating Engineering Society (IES), also proposed by Reiner [10]. By applying texture, our goal was to reproduce the brushing pattern introduced by industrial process. The texture is an important realism property, therefore our aim is to investigate different texture synthesis methods. Acquisition system enabled comparison of synthesized and real image satisfying requirement R3. As it can be seen in Fig. 7, without hand-eye calibration a miss-match in the overlap of the object in the rendered and the real image is present. This mismatch has been removed using the handeye calibration (see Fig. 7). Matching also depends on the distortion parameters of the camera lens. Currently, calibration system can only model radial distortion and not tangential distortion. Effects of tangential distortion can be seen in the lower right half of the image. The effect of tangential distortion increases as we move away from image center. Modeling tangential distortion will further improve the results. As it can be seen, the presented pipeline satisfies all requirements discussed in section III, also it provides clear steps as well as general solution for image synthesis for surface inspection.

\section{CONCLUSION}

The lack of images containing defects is a common obstacle in industrial inspection. In this paper, we have presented a pipeline for synthesizing images of an object with both ideal and defective surfaces. It consists of three modules. The first module, Errsmith, is responsible for imprinting the object geometry with surface defects of various sizes and positions. This approach ensures valid light response around defective areas of the surface, which seems impossible when augmenting defects from real images to defect-free images. The second module, Callistemon, performs realistic image synthesis which incorporates surface inspection data along with material, light, and camera specification to ensure realistic appearance. The third module provides the acquisition of images through a robotic system and ensures that the geometry of the objects in synthesized images and real images match. This pipeline promises numerous applications. One example is synthesizing images containing features unseen in real-world data for machine learning approaches. It can be also used for simulating inspection systems while designing specialized hand-crafted algorithms. Also, it can help during viewpoint optimization for surface coverage where it is required to know if a certain defect is visible from a particular camera and light position and orientation. For further work, we would point out the importance of automatic evaluation of the synthesized image with regards to the real image and the metric which would allow this. If synthesizing images containing features unseen in real data would help defect detection models learn to detect surface defects, could inspection systems start developing in similar directions?

\section{ACKNOWLEDGMENT}

Authors would like to thank for the support from Fraunhofer ITWM-BV. 


\section{REFERENCES}

[1] P. Gospodnetic, D. Mosbach, M. Rauhut, and H. Hagen, "Flexible surface inspection planning pipeline," in 2020 6th International Conference on Control, Automation and Robotics (ICCAR), 2020.

[2] M. Mohammadikaji, "Simulation-based planning of machine vision inspection systems with an application to laser triangulation," Ph.D. dissertation, KIT, 2019.

[3] C. Shorten and T. Khoshgoftaar, "A survey on image data augmentation for deep learning," Journal of Big Data, vol. 6, 122019.

[4] W. Tang, Q. Yang, K. Xiong, and W. Yan, "Deep learning based automatic defect identification of photovoltaic module using electroluminescence images," Solar Energy, vol. 201, pp. 453 - 460, 2020.

[5] S. Mérillou, J.-M. Dischler, and D. Ghazanfarpour, "Surface scratches: measuring, modeling and rendering," The Visual Computer, vol. 17, pp. 30-45, 2001.

[6] B. Desbenoit, E. Galin, and S. Akkouche, "Modeling cracks and fractures," The Visual Computer, vol. 21, pp. 717-726, 2005.

[7] M. Haindl and J. Filip, Visual texture: Accurate material appearance measurement, representation and modeling. Springer Science \& Business Media, 2013.

[8] A. Ngan, F. Durand, and W. Matusik, "Experimental analysis of brdf models." Rendering Techniques, vol. 2005, no. 16th, p. 2, 2005.

[9] B. Walter, S. R. Marschner, H. Li, and K. E. Torrance, "Microfacet models for refraction through rough surfaces." Rendering techniques, vol. 2007, p. 18th, 2007.

[10] J. Reiner, "Rendering for machine vision prototyping," in Optical Design and Engineering III, vol. 7100. International Society for Optics and Photonics, 2008, p. 710009.

[11] Z. Dong, B. Walter, S. Marschner, and D. P. Greenberg, "Predicting appearance from measured microgeometry of metal surfaces," $A C M$ Transactions on Graphics (TOG), vol. 35, no. 1, pp. 1-13, 2015.

[12] H. Kolivand, M. S. Sunar, S. Y. Kakh, R. Al-Rousan, and I. Ismail, "Photorealistic rendering: a survey on evaluation," Multimedia Tools and Applications, vol. 77, no. 19, pp. 25 983-26008, 2018.

[13] D. P. Greenberg, K. E. Torrance, P. Shirley, J. Arvo, E. Lafortune, J. A. Ferwerda, B. Walter, B. Trumbore, S. Pattanaik, and S.-C. Foo, "A framework for realistic image synthesis," in Proceedings of the 24th annual conference on Computer graphics and interactive techniques, 1997, pp. 477-494.

[14] J. D. Stets, A. D. Corso, J. B. Nielsen, R. A. Lyngby, S. H. N. Jensen, J. Wilm, M. B. Doest, C. Gundlach, E. R. Eiriksson, K. Conradsen, A. B. Dahl, J. A. Bærentzen, J. R. Frisvad, and H. Aanæs, "Scene reassembly after multimodal digitization and pipeline evaluation using photorealistic rendering," Appl. Opt., vol. 56, no. 27, pp. 7679-7690, Sep 2017. [Online]. Available: http://ao.osa.org/abstract.cfm?URI=ao56-27-7679

[15] R. Y. Tsai and R. K. Lenz, "A new technique for fully autonomous and efficient $3 \mathrm{~d}$ robotics hand/eye calibration," IEEE Transactions on robotics and automation, vol. 5, no. 3, pp. 345-358, 1989.

[16] K. Pachtrachai, M. Allan, V. Pawar, S. Hailes, and D. Stoyanov, "Handeye calibration for robotic assisted minimally invasive surgery without a calibration object," in 2016 IEEE/RSJ International Conference on Intelligent Robots and Systems (IROS). IEEE, 2016, pp. 2485-2491.

[17] M. Antonello, A. Gobbi, S. Michieletto, S. Ghidoni, and E. Menegatti, "A fully automatic hand-eye calibration system," 09 2017, pp. 1-6.

[18] H. Wang, X. Lu, Z. Hu, and Y. Li, "A vision-based fully-automatic calibration method for hand-eye serial robot," Industrial Robot: An International Journal, 2015.

[19] R. A. Lyngby, J. B. Matthiassen, J. R. Frisvad, A. B. Dahl, and H. Aanæs, "Using a robotic arm for measuring brdfs," in Scandinavian Conference on Image Analysis. Springer, 2019, pp. 184-196.

[20] R. Liang and J. Mao, "Hand-eye calibration with a new linear decomposition algorithm," Journal of Zhejiang University-SCIENCE A, vol. 9, no. 10, pp. 1363-1368, 2008.

[21] J. Dorsey, H. Rushmeier, and F. Sillion, Digital modeling of material appearance. Elsevier, 2010.

[22] F. Beaune, E. Tovagliari, L. Barrancos, S. Agyemang, S. Basu, M. Bhutani, L. Bosnar, R. Brune, M. Chan, J. M. M. Costa, H. Crepaz, J. Deng, J. Dent, M. Dhiman, D. Fevrier, K. R. Iyer, D. Lahiri, K. Masson, G. Olson, A. Pandey, J. Park, S. Pogosyan, B. Samir, O. Smolin, T. Vergne, L. Wilimitis, and L. Zawallich, "appleseed," Sep. 2019. [Online]. Available: https://doi.org/10.5281/zenodo.3456967
[23] M. Pharr, W. Jakob, and G. Humphreys, Physically based rendering: From theory to implementation. Morgan Kaufmann, 2016.

[24] W. McDermott, The PBR Guide. Allegorithmic, 2018.

[25] L. Gritz, C. Stein, C. Kulla, and A. Conty, "Open shading language," in ACM SIGGRAPH 2010 Talks, 2010, pp. 1-1.

[26] G. Bradski, "The OpenCV Library," Dr. Dobb's Journal of Software Tools, 2000.

[27] É. Marchand, F. Spindler, and F. Chaumette, "Visp for visual servoing: a generic software platform with a wide class of robot control skills," IEEE Robotics \& Automation Magazine, vol. 12, no. 4, pp. 40-52, 2005. 